\title{
Long noncoding RNA myocardial infarction-associated transcript is associated with the microRNA-150-5p/P300 pathway in cardiac hypertrophy
}

\author{
ZHAO LI ${ }^{1 *}$, YAMIN LIU ${ }^{2 *}$, XIAOFAN GUO ${ }^{1}$, GUOZHE SUN $^{1}$, QUN MA ${ }^{1}$, \\ YING DAI ${ }^{3}$, GUANGSHUO ZHU ${ }^{3}$ and YINGXIAN SUN ${ }^{1}$ \\ ${ }^{1}$ Department of Cardiology, The First Hospital of China Medical University, Shenyang, \\ Liaoning 110001; ${ }^{2}$ Department of Pharmacy, Zhongda Hospital, Southeast University, Nanjing, \\ Jiangsu 210009, P.R. China; ${ }^{3}$ Department of Cardiology, Johns Hopkins University, Baltimore, MD 21205, USA
}

Received November 5, 2017; Accepted May 17, 2018

DOI: $10.3892 / \mathrm{ijmm} .2018 .3700$

\begin{abstract}
In numerous diseases, abnormal expression of myocardial infarction-associated transcript (MIAT) has been reported to be involved in cell proliferation, apoptosis and migration. However, whether this long non-coding RNA MIAT has a regulatory effect on heart hypertrophy requires further investigation. To this end, the present study evaluated MIAT in hypertrophic cardiomyocytes in vitro and in vivo. Neonatal rat ventricular myocytes (NRVMs) were induced by isoproterenol (ISO) to create a cell hypertrophy model, and mice were intraperitoneally injected with ISO to establish an animal model. Echocardiography, immunofluorescence staining, western blot analysis, RNA isolation and reverse transcription-polymerase chain reaction were applied to test the involvement of MIAT in cardiac hypertrophy. The results revealed that MIAT was upregulated under ISO stimulation at the mRNA level both in vivo and in vitro. Silencing of MIAT resulted in decreased expression levels of atrial natriuretic peptide and brain natriuretic peptide in ISO-treated NRVM cardiomyocytes, confirming the connection between MIAT and hypertrophy. Furthermore, MIAT small interfering RNA significantly increased microRNA (miR)-150 and decreased P300 expression in NRVMs. In conclusion, the MIAT/miR-150-5p axis targets $\mathrm{P} 300$ as a positive regulator of cardiomyocyte hypertrophy.
\end{abstract}

Correspondence to: Dr Yingxian Sun, Department of Cardiology, The First Hospital of China Medical University, 155 Nanjing North Street, Heping, Shenyang, Liaoning 110001, P.R. China

E-mail: sunyingxian121@126.com

${ }^{*}$ Contributed equally

Key words: myocardial infarction-associated transcript, neonatal rat ventricular myocytes, hypertrophy, microRNA-150, P300

\section{Introduction}

Previous studies have reported that protein-coding genes account for only a small proportion of the genome, while the larger remaining part includes genes that are either not transcribed or are transcribed as non-coding RNA (1,2). Currently, the function of non-coding RNA merits further investigation. Generally, long non-coding RNAs (lncRNAs) do not encode proteins and have a length of at least 200 nucleotides (3). Research has confirmed the involvement of lncRNAs in the process of transcription and post-transcription in tumor cells (4-6); however, the mechanisms of IncRNA action in myocytes have received relatively limited attention.

In 2006, myocardial infarction-associated transcript (MIAT) was identified as an lncRNAs that confers a risk of myocardial infraction (7). MIAT is also known as retinal non-coding RNA 2, AK028326 or Gomafu (8). Subsequent studies have reported that MIAT participates in a variety of diseases and cellular processes, including myocardial infarction, microvascular dysfunction (9), paranoid schizophrenia (10), neurogenic commitment (11) and the formation of nuclear bodies (12). However, only a limited number of studies have examined MIAT in myocyte hypertrophy $(13,14)$. MIAT was reported to suppress microRNA (miR)-150 expression in cardiomyocytes (14). Furthermore, previous studies have demonstrated that cardiomyocyte hypertrophy is modulated by miR-150 via its regulatory effect on the expression of the transcriptional co-activator P300 (15). However, it remains unclear whether MIAT is involved in the pathway of miR-150-5p/P300. Therefore, the present study investigated the involvement of MIAT in hypertrophy in vitro and in vivo through cell and animal models.

\section{Materials and methods}

Animal care and use. This study conformed to the Guide for the Care and Use of Laboratory Animals as published by the National Institutes of Health (NIH publication no. 85-23; revised 1996). All procedures were performed in accordance with the Ethical Standards of the Committee 
on Animal Experimentation of China Medical University (project identification code, SCXK-2013-0001; Shenyang, China), and were approved by the Ethical Standards of the Committee on Animal Experimentation of the First Hospital of China Medical University (no. 2017010). Healthy male specific-pathogen-free C57BL/6J mice (age, 8 weeks; weight, 19-21 g) were purchased from the Laboratory Animal Center of China Medical University, and cared for as reported previously (16). Mice were kept in a $12 \mathrm{~h}$ light-dark cycle, temperature-controlled $\left(22 \pm 2^{\circ} \mathrm{C}\right)$ and humidity-controlled $(55 \pm 5 \%)$ environment, and fed a standard chow diet for at least 1 week before experimentation.

Animal procedures and echocardiography. Mice were randomized into two groups, and intraperitoneally injected with $20 \mu \mathrm{g} / \mathrm{kg}$ of isoproterenol (ISO; diluted with $0.9 \%$ $\mathrm{NaCl} ; \mathrm{n}=4)$ or vehicle $(0.9 \% \mathrm{NaCl} ; \mathrm{n}=4)$ twice a day for 7 days. Transthoracic echocardiography was performed in conscious mice under isofluorane anesthesia (1\% isoflurane; 0.6-liter flow of $\mathrm{O}_{2}$ ) using a Vevo 2100 imaging system (FUJIFILM VisualSonics Inc., Toronto, Canada). B and $\mathrm{M}$-mode images were obtained, and other data were collected from each mouse group prior to and following the injection week. For obtaining the left ventricular images, the cursor was positioned at the level just posterior to the chordae tendinae, perpendicular to the interventricular septum and left ventricular posterior wall. Left ventricular posterior wall dimensions at diastole (LVPWd) and interventricular septal thickness at diastole (IVSd) were measured from the left ventricle-left ventricular internal dimension at diastole.

The mice were then sacrificed and weighed, subsequently the hearts were harvested, rinsed and weighted, and the tibias were measured. Ventricles were either stored in TRIzol reagent (Thermo Fisher Scientific, Inc., Waltham, MA, USA) for RNA extraction or radio immunoprecipitation assay lysis buffer (RIPA) for protein extraction.

Histological analyses. Excised mice hearts were immediately fixed in $10 \%$ neutral buffered formalin for $24 \mathrm{~h}$ at room temperature after a brief rinse with cold PBS, followed by dehydration and embedding in paraffin wax. Left ventricular sections $(4 \mu \mathrm{m})$ were cut and stained with hematoxylin and eosin (H\&E) to measure cardiomyocyte cross-sectional area. Briefly, 4- $\mu \mathrm{m}$ microtome sections were deparaffinized with xylene and rehydrated through graded concentrations of ethanol (100, 95 and 80\%), followed by staining with hematoxylin for $4 \mathrm{~min}$ at room temperature. Subsequently, slides were rinsed in deionized water and dipped in $0.25 \%$ acidic ethanol for $2 \mathrm{sec}$. Following washing with deionized water, the slides were immersed in eosin for $2 \mathrm{~min}$ at room temperature. Slides were then rinsed thoroughly in three changes of absolute alcohol. Finally, the slides were washed with xylene and then mounted in Permount.

Histology images were obtained with a Leica DM6000 microscope and a DFC420 C camera (Leica Microsystems $\mathrm{GmbH}$, Wetzlar, Germany; magnifications, x10 and 40). The cardiomyocyte cross-sectional area per nucleus was measured. The selection of cardiomyocytes for measurement was based on clearly defined sarcolemma and associated round nuclei.
Cross-sectional areas in over 200 randomly chosen cardiomyocytes from at least 16 fields per group were evaluated using ImageJ software (version 1.48, National Institutes of Health, Bethesda, MD, USA).

Primary culture of neonatal rat ventricular myocytes (NRVMs) and treatment. NRVMs were isolated as previously described (17). Briefly, newborn Sprague-Dawley rats (1-3-days-old) were sacrificed, their hearts were removed and the atria were immediately separated. Cold Hank's equilibrium salt solution with no $\mathrm{Ca}^{2+}$ and $\mathrm{Mg}^{2+}$ (Gibco; Thermo Fisher Scientific, Inc.) was used to store the ventricle tissues. Subsequent to transferring the ventricles to trypsin- $0.125 \%$ EDTA (Invitrogen; Thermo Fisher Scientific, Inc.) and storing overnight at $4^{\circ} \mathrm{C}(16-20 \mathrm{~h})$, collagenase II (Worthington Biochemical Corporation, Lakewood, NJ, USA) was used for further digestion at $37^{\circ} \mathrm{C}$ for $30 \mathrm{~min}$ in a shaking water bath. Isolated cells were prepared in an uncoated flask at $37^{\circ} \mathrm{C}$ to remove cardiac fibroblasts, and cells were harvested from the supernatants after $1.5 \mathrm{~h}$. NRVMs were seeded in plating medium containing 45\% Dulbecco's modified Eagle's medium (DMEM), 45\% M199 medium, 10\% fetal bovine serum, $100 \mathrm{U} / \mathrm{ml}$ penicillin and $100 \mu \mathrm{g} / \mathrm{ml}$ streptomycin (all obtained from Thermo Fisher Scientific, Inc.) for $24 \mathrm{~h}$ at $37^{\circ} \mathrm{C}$. The following day, the plating medium was replaced by maintenance medium containing 50\% DMEM, 50\% M199 without serum, $100 \mathrm{U} / \mathrm{ml}$ penicillin and $100 \mu \mathrm{g} / \mathrm{ml}$ streptomycin, and incubated for an additional $24 \mathrm{~h}$ at $37^{\circ} \mathrm{C}$ prior to being used for experimentation. NRVMs were incubated with or without $10 \mu \mathrm{M}$ ISO for $24 \mathrm{~h}$.

Cell transfection. MIAT siRNA (si-MIAT) and the negative control siRNA (si-NC) were purchased from GenePharma Co., Ltd. (Shanghai, China). Their sequences were as follows: Negative control siRNA, 5'-UUCUCCGAACGUGUCACG UTT-3' (sense) and 5'-ACGUGACACGUUCGGAGAATT-3' (antisense); MIAT siRNA, 5'-GGUGUUAAGACUUGGUUU CTT-3' (sense) and 5'-GAAACCAAGUCUUAACACCTT-3' (antisense). NRVMs were transfected with $100 \mathrm{nM}$ MIAT siRNA or negative control for $6 \mathrm{~h}$ using Lipofectamine ${ }^{\circledR} 3000$ (Invitrogen; Thermo Fisher Scientific, Inc.) according to manufacturer instructions. Briefly, NRVMs were seeded into a 6 -well plate at $6 \times 10^{5}$ cells per well in $2 \mathrm{ml}$ plating medium for $24 \mathrm{~h}$ at $37^{\circ} \mathrm{C}$, and then incubated in serum-free maintenance medium for at least $24 \mathrm{~h}$. When $60-70 \%$ confluence was reached, transfected NRVMs were then treated with or without ISO $(10 \mu \mathrm{m})$ for $48 \mathrm{~h}$ at $37^{\circ} \mathrm{C}$. Cells were collected after $48 \mathrm{~h}$ for quantification of mRNA and miRNA expression.

Immunofluorescence staining and measurement of NRVM cell area. NRVMs were stained with phalloidin conjugated with Alexa Fluor 594, and nuclei were stained with DAPI (all from Invitrogen; Thermo Fisher Scientific, Inc.). Slides were visualized using an Olympus AX70 Provis fluorescence microscope at a 40 magnification (Olympus Corporation, Tokyo, Japan). In each group, 30 cells were counted, and the fields-of-view of cells were randomly selected under each condition. The surface area was estimated by the ratio of control cells. Each experiment was repeated in triplicate. 
$R N A$ isolation and reverse transcription-quantitative polymerase chain reaction ( $R T-q P C R)$. Total RNA from the tissue and NRVMs was extracted using TRIzol reagent (Thermo Fisher Scientific, Inc.) according to manufacturer's protocol. RNAs with an OD260/OD280 ratio in the range of 1.80-2.20 were used for downstream assays, and $1 \mu \mathrm{g}$ RNA from each sample was reverse transcribed into cDNA using a high-capacity cDNA RT kit (Applied Biosystems; Thermo Fisher Scientific, Inc.). The RT conditions were conducted as per the manufacturer's protocols. Subsequently, qPCR was performed to quantify the mRNA expression levels using Real-Time SYBR ${ }^{\circledR}$ Green PCR Master Mix (Bio-Rad Laboratories, Inc., Hercules, CA, USA) on the CFX384 Touch Real-Time PCR Detection system (Bio-Rad Laboratories, Inc.), with GAPDH serving as an internal control. Expression levels were analyzed using the $2^{-\Delta \Delta \mathrm{Cq}}$ method (18). Primers sequences used in the project are listed in Table I.

For miRNA analysis, cDNA was synthesized using the TaqMan $^{\mathrm{TM}}$ MicroRNA Reverse Transcription kit (Applied Biosystems; Thermo Fisher Scientific, Inc.). The miR-150-5p expression were determined by the TaqMan ${ }^{\mathrm{TM}}$ MicroRNA assay (cat. no. A25576, Applied Biosystems; Thermo Fisher Scientific, Inc.) and Taqman $2 X$ universal PCR master mix (cat. no. 4304437, Applied Biosystems; Thermo Fisher Scientific, Inc.) on the CFX384 Touch Real-Time PCR Detection System (Bio-Rad Laboratories, Inc.), with U6 small nuclear RNA (U6) assay (cat. no. 4427975, Applied Biosystems; Thermo Fisher Scientific, Inc.) serving as an internal control. Expression levels were analyzed using the $2^{-\Delta \Delta \mathrm{Cq}}$ method (18).

Western blot analysis. Samples from mice and NRVMs were lysed in radio immunoprecipitation assay lysis buffer (RIPA, cat. no. 9806, Cell Signaling Technology, Inc., Danvers, MA, USA) with protease inhibitors. Protein concentrations were then quantified using a bicinchoninic acid assay kit (Thermo Fisher Scientific, Inc.). Cell lysate ( $20 \mu \mathrm{g}$ protein) was isolated with $10 \%$ sodium dodecyl sulfate-polyacrylamide gel electrophoresis, and the protein was transferred to polyvinylidene difluoride membranes. The membranes were subsequently blocked using 5\% BSA for $1 \mathrm{~h}$ at room temperature, and incubated with atrial natriuretic peptide (ANP) primary antibody (cat. no. ab189921; 1:2,000; Abcam, Cambridge, MA, USA) overnight at $4^{\circ} \mathrm{C}$. The membrane was also incubated with GAPDH (cat. no. 5174; 1:20,000; Cell Signaling Technology, Inc.) for $1 \mathrm{~h}$ at room temperature. Subsequently, a horseradish peroxidase-linked anti-rabbit $\mathrm{IgG}$ secondary antibody (cat. no. 7074; 1:20,000; Cell Signaling Technology, Inc.) was then used incubated for $1 \mathrm{~h}$ at room temperature. An enhanced chemiluminescence reagent (SuperSignal ${ }^{\mathrm{TM}}$ West Femto Maximum Sensitivity Substrate; Thermo Fisher Scientific, Inc.) was used to visualize the bands, and semi-quantification of western blot bands was conducted by comparison against the GAPDH bands using ImageJ software (version 1.48, National Institutes of Health, Bethesda, MD, USA).

Statistical analysis. Data are reported as the mean \pm standard error. Comparisons between groups were analyzed by double-sided Student's t-test, or with analysis of variance and Turkey's test for experiments with more than two subgroups. $\mathrm{P}<0.05$ was considered to indicate a statistically significant
Table I. Sequences of polymerase chain reaction primers.

Target ID Sequence

GAPDH Forward: 5'-AGTGCCAGCCTCGTCTCATA-3' Reverse: 5'-GGTAACCAGGCGTCCGATAC-3

MIAT Forward: 5'-TCTCTGGTGCTTCCCTCCTT-3' Reverse: 5'-GATCTAAGCTTGAGCCCCCA-3'

ANP Forward: 5'-CTCCCAGGCCATATTGGAG-3' Reverse: 5'-TCCAGGTGGTCTAGCAGGTT-3'

BNP Forward: 5'-GAGTCCTTCGGTCTCAAGGC-3' Reverse: 5'-CAACTTCAGTGCGTTACAGCC-3'

P300 Forward:5'-GACCCTCAGCTTTTAGGAATCC-3' Reverse: 5'-TGCCGTAGCAACACAGTGTCT-3'

difference. Statistical analysis was performed using IBM SPSS software (version 24; IBM Corp., Armonk, NY, USA).

\section{Results}

MIAT, miR-150-5p and P300 expression levels are associated with cardiomyocyte hypertrophy induced by ISO in vivo. ISO was injected in mice in order to build a cardiac hypertrophy model. Mice were injected $20 \mathrm{mg}$ ISO/kg twice per day over 7 days, and cardiac hypertrophy in the ISO and control group mice was analyzed. Echocardiography, histopathology and morphometric analysis were conducted to confirm that ISO induced cardiac hypertrophy. Firstly, we performed echocardiography to measure the cardiac parameters in B and M modes after 7 day's ISO injection. The IVSd and LVPWd in ISO mice were significantly higher than in control mice $(0.843 \pm 0.03$ vs. $0.69 \pm 0.19, \mathrm{P}<0.05 ; 0.80 \pm 0.26$ vs. $0.63 \pm 0.21$, $\mathrm{P}<0.05$; respectively; Fig. $1 \mathrm{~A}$ and $\mathrm{B})$. In addition, heart anatomy of the ISO and control mice was conducted. As presented in Table II, after the heart weight (HW) was normalized to tibia length (TL), a higher HW/TL ratio was observed in ISO mice than that of control mice $(0.083 \pm 0.007$ vs. $0.108 \pm 0.0014, \mathrm{P}<0.05)$. Furthermore, histological analysis revealed that cardiomyocyte cross-sectional area was significantly increased in ISO-induced group compared with the control group $(1 \pm 0.08$ vs. $2.6 \pm 0.81, \mathrm{P}<0.05$, respectively; Fig. 2A and B).

To investigate whether MIAT and miR-150-5p were involved in the development of cardiac hypertrophy in vivo, MIAT and miR-150-5p expression levels in heart tissue were assessed by RT-qPCR. As shown in Fig. 3, the expression levels of ANP and BNP were higher in the ISO group (Fig. 3A). The protein expression levels of ANP were also analyzed, which revealed a significant increase in the ISO group compared with in the control group (Fig. 3B). Additionally, MIAT expression levels were significantly increased in the ISO group compared with control groups (Fig. 3C). miR-150 expression levels were significantly decreased in ISO group compared with control group (Fig. 3D). Conversely, the expression levels of P300 in the ISO group were $\sim 5$-fold higher in comparison with that of the control group (Fig. 3E). The results of the present study suggested that MIAT, miR-150-5p and P300 may be involved 
Table II. Morphometric analysis.

\begin{tabular}{lrrr}
\hline Parameter & \multicolumn{1}{c}{ Con } & \multicolumn{1}{c}{ ISO } & P-value \\
\hline BW (g) & $27.75000 \pm 0.89000$ & $26.75000 \pm 0.61000$ & 0.78000 \\
HW (mg) & $149.00000 \pm 1.30000$ & $192.00000 \pm 2.10000$ & 0.03800 \\
HW/BW & $0.00530 \pm 0.00043$ & $0.00729 \pm 0.00010$ & 0.01900 \\
TL (mm) & $17.80000 \pm 0.20000$ & $17.80000 \pm 0.15000$ & 0.95000 \\
HW/TL & $0.08300 \pm 0.00700$ & $0.10800 \pm 0.00140$ & 0.03260
\end{tabular}

BW, body weight; HW, heart weight; TL, tibial length.

A
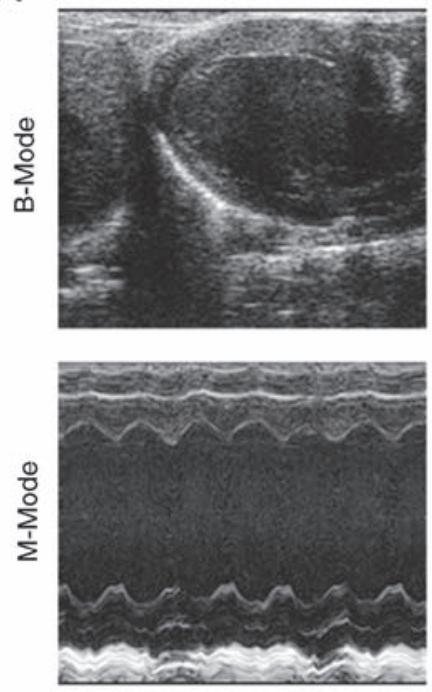

ISO
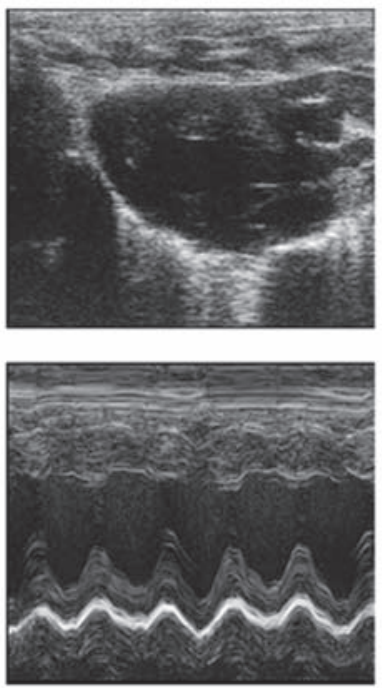

B

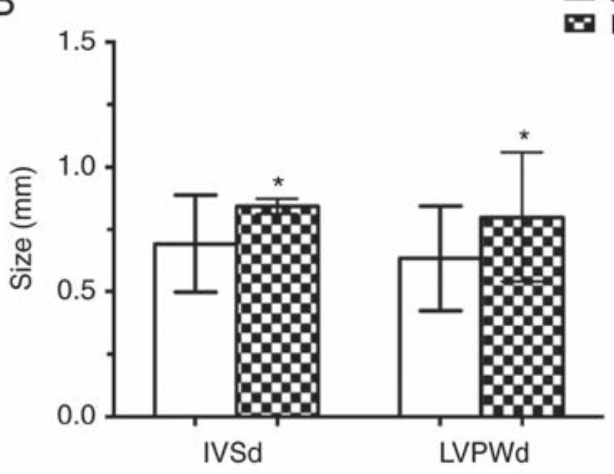

Figure 1. ISO induced cardiac hypertrophy, as determined by echocardiography. (A) Representative echocardiography images of the Con (left) and ISO (right) groups in B- and M-modes. (B) Cardiac parameters of mouse model detected by M-mode echocardiography. LVPWd and IVSd of the Con and ISO groups $(\mathrm{n}=4)$ are shown. "P<0.05 vs. Con group. ISO, isoproterenol; Con, control; LVPWd, left ventricular posterior wall thickness at end diastole; IVSd, interventricular septal end-diastole.

A
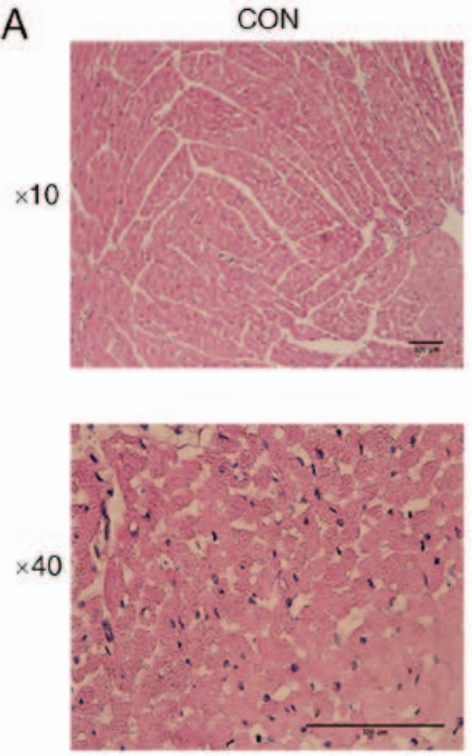

ISO
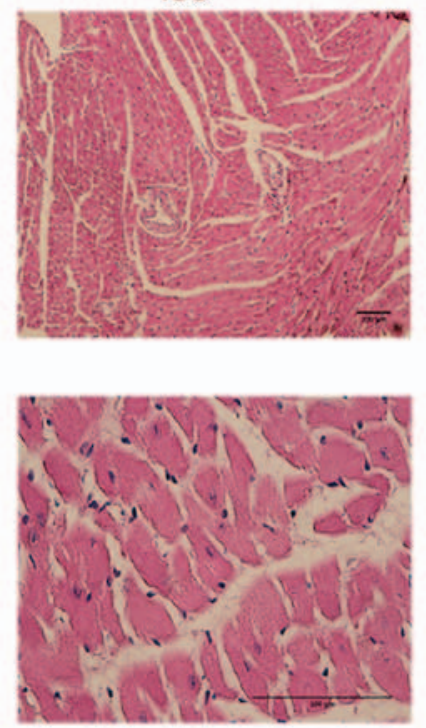

B

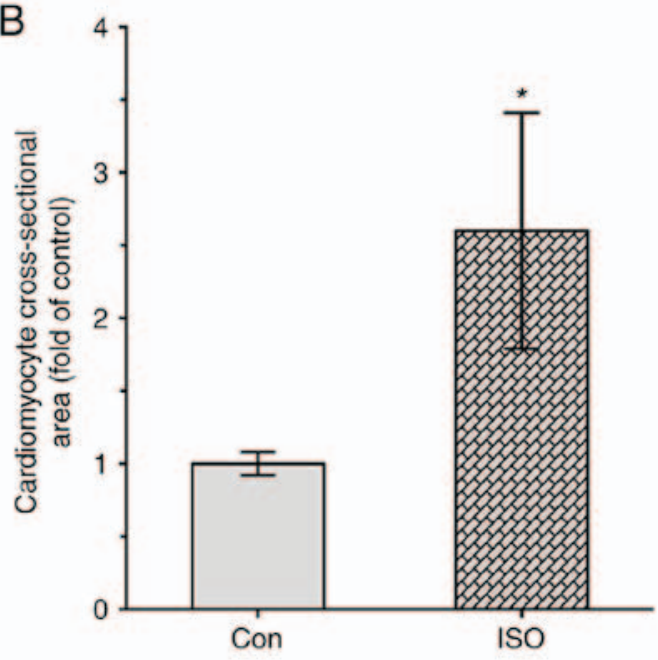

Figure 2. ISO-induced cardiac hypertrophy. Mice were injected with $20 \mathrm{mg} / \mathrm{kg}$ ISO twice per day for 7 days. (A) Transverse sections from the left ventricle of control mice and ISO mice were stained with H\&E. Panel A top and bottom: Magnifications, x10 and 40, respectively. Scale bar=100 $\mu$ m. (B) Cardiomycytes cross-sectional area were increased in ISO group compared with in control mice. At least 200 cells in each group (n=4). $\mathrm{P}<0.05$ vs. Con group. ISO, isoproterenol; Con, control. 
A


B
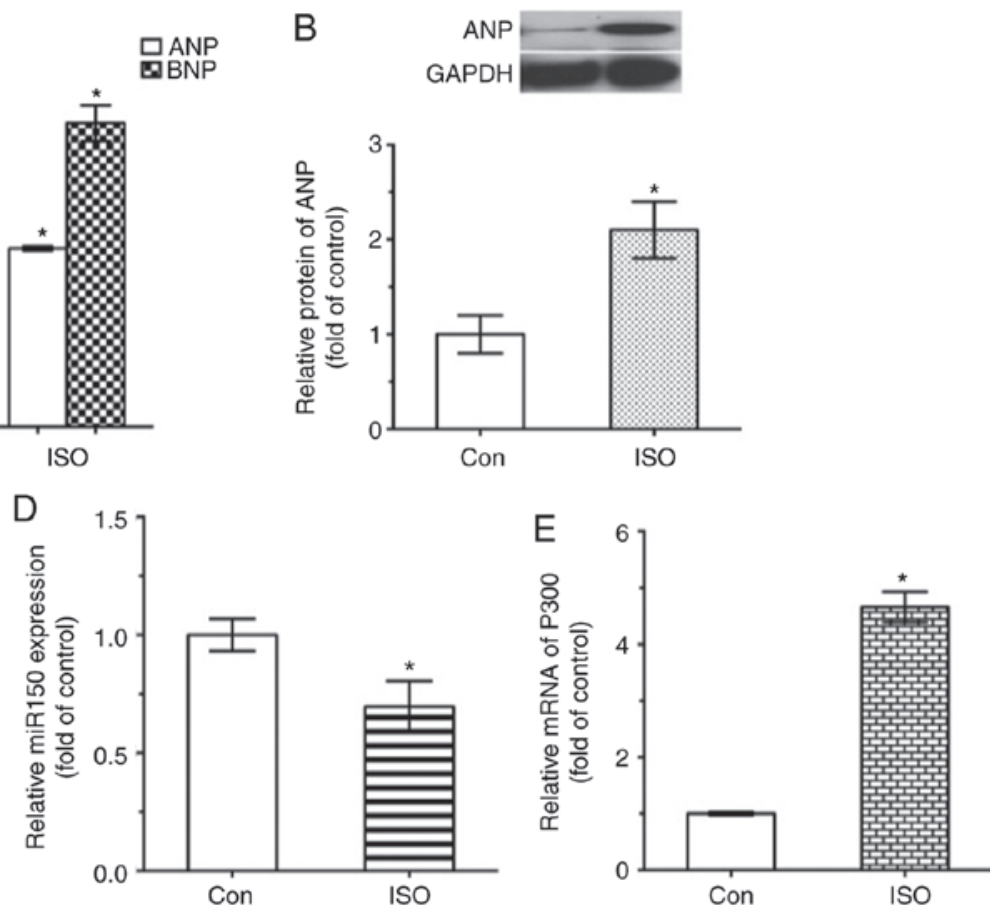

Figure 3. Expression levels of MIAT, miR-150-5p and P300 in ISO-induced hypertrophy mouse model. mRNA and protein levels were analyzed by reverse transcription-quantitative polymerase chain reaction and western blot analysis, respectively. (A) ANP and BNP mRNA, (B) ANP protein, (C) MIAT mRNA, (D) miR-150-5p and (E) P300 mRNA expression levels are presented. ${ }^{*} \mathrm{P}<0.05$ vs. control group ( $\mathrm{n}=4$ ). ANP, atrial natriuretic peptide; BNP, brain natriuretic peptide; Con, control; ISO, isoproterenol; MIAT, myocardial infarction-associated transcript; miR, microRNA.

in the hypertrophic process of myocytes and affect the cardiac function in vivo.

MIAT, miR-150-5p, and P300 expression is associated with cardiomyocyte hypertrophy induced by ISO in vitro. Since ISO was observed to lead to myocyte hypertrophy in vivo, the effect of ISO in NRVMs was also examined in vitro. NRVMs were incubated for $24 \mathrm{~h}$ in the presence or absence of $10 \mu \mathrm{M}$ ISO, and their surface areas were then measured. As shown in Fig. 4, the myocyte size was increased in the group treated with ISO compared with that in the control group. Furthermore, the expression levels of ANP and BNP, biomarkers of cardiomyocyte hypertrophy, were observed to be significantly upregulate in the ISO group by using RT-qPCR and western blotting (Fig. 5A and B).

Subsequently, MIAT expression was assessed in ISO-treated NRVMs. As shown in Fig. 5, it was determined that the level of MIAT was significantly increased in cardiomyocytes with ISO-induced hypertrophy, suggesting that MIAT was associated with hypertrophy (Fig. 5C). Furthermore, miR-150-5p expression was also assessed, and it was observed that miR-150-5p was markedly decreased in ISO-induced NRVMs (Fig. 5D). Additionally, compared with in the control group, P300 expression levels were significantly increased in NRVMs treated with ISO (Fig. 5E). These results suggested that MIAT, miR-150-5p, and P300 expression may be associated with cardiomyocyte hypertrophy induced by ISO in vitro.

MIAT may upregulate P300 expression by suppression of $m i R-150-5 p$. Since MIAT and miR-150-5p were observed to be connected with hypertrophy in the aforementioned results,

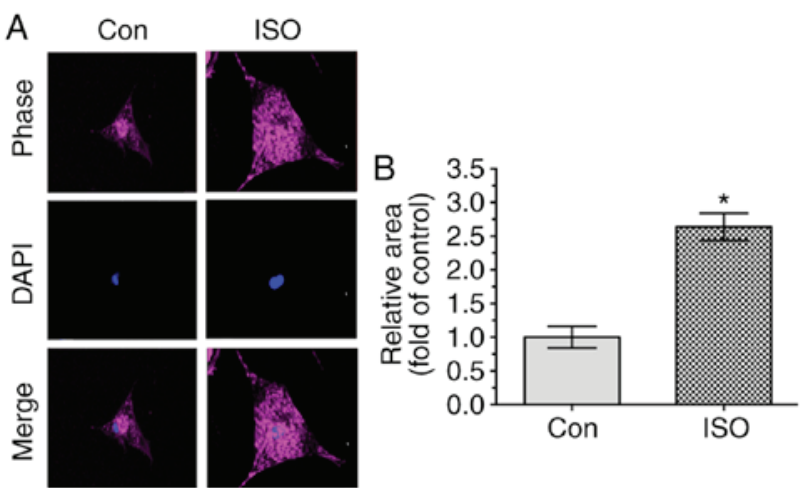

Figure 4. ISO induced hypertrophy in NRVMs. (A) Morphology of NRVMs in the presence or absence of $10 \mu \mathrm{M}$ ISO for $24 \mathrm{~h}$. The cellular actin was stained with phalloidin conjugated with Alexa Fluor 594 (upper panels). Nuclei were dyed blue using $300 \mathrm{nM}$ DAPI (middle panels). Merged images are shown in the lower panels (bars $=20 \mu \mathrm{m}$ ). (B) Cell surface area changes were measured with a quantitative digital image analysis system. ${ }^{*} \mathrm{P}<0.05$ vs. Con group ( $n=3)$. ISO, isoproterenol; Con, control; NRVMs, neonatal rat ventricular myocytes.

it was then hypothesized that the pathological effect of MIAT in cardiac hypertrophy may be associated with miR-150-5p. To test this hypothesis, we measured ANP, BNP, P300 and miR-150 expression in NRVMs by RT-qPCR after transfecting NRVMs with si-MIAT (Fig. 6). The results revealed that MIAT expression levels were downregulated in NRVMs following transfection with si-MIAT for $48 \mathrm{~h}$ (Fig. 6A). Furthermore, the NRVMs were treated with or without ISO following transfection with si-NC or si-MIAT, which demonstrated a significant decrease in the expression levels of ANP and BNP within si-MIAT cells than that of si-NC cells (Fig. 6B). In addition, 

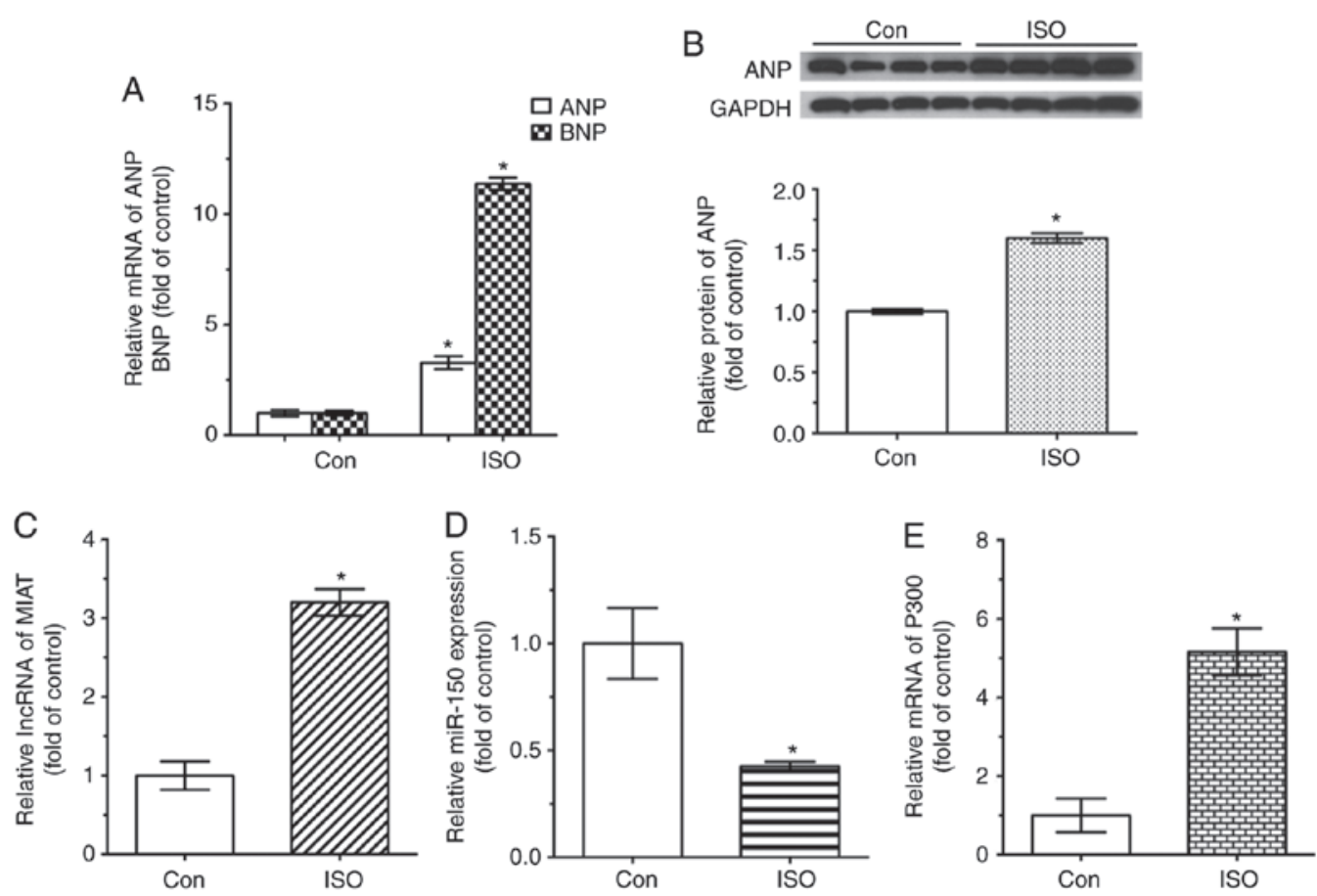

Figure 5. Expression levels of MIAT, miR-150-5p and P300 in NRVMs induced by ISO. mRNA and protein levels were analyzed by reverse transcriptionquantitative polymerase chain reaction and western blot analysis, respectively. (A) ANP and BNP mRNA, (B) ANP protein, (C) MIAT mRNA, (D) miR-150-5p and (E) P300 mRNA expression levels are shown. ${ }^{*} \mathrm{P}<0.05$ vs. Con group $(\mathrm{n}=3)$. ANP, atrial natriuretic peptide; BNP, brain natriuretic peptide; ISO, isoproterenol; Con, control; MIAT, myocardial infarction-associated transcript; miR, microRNA.
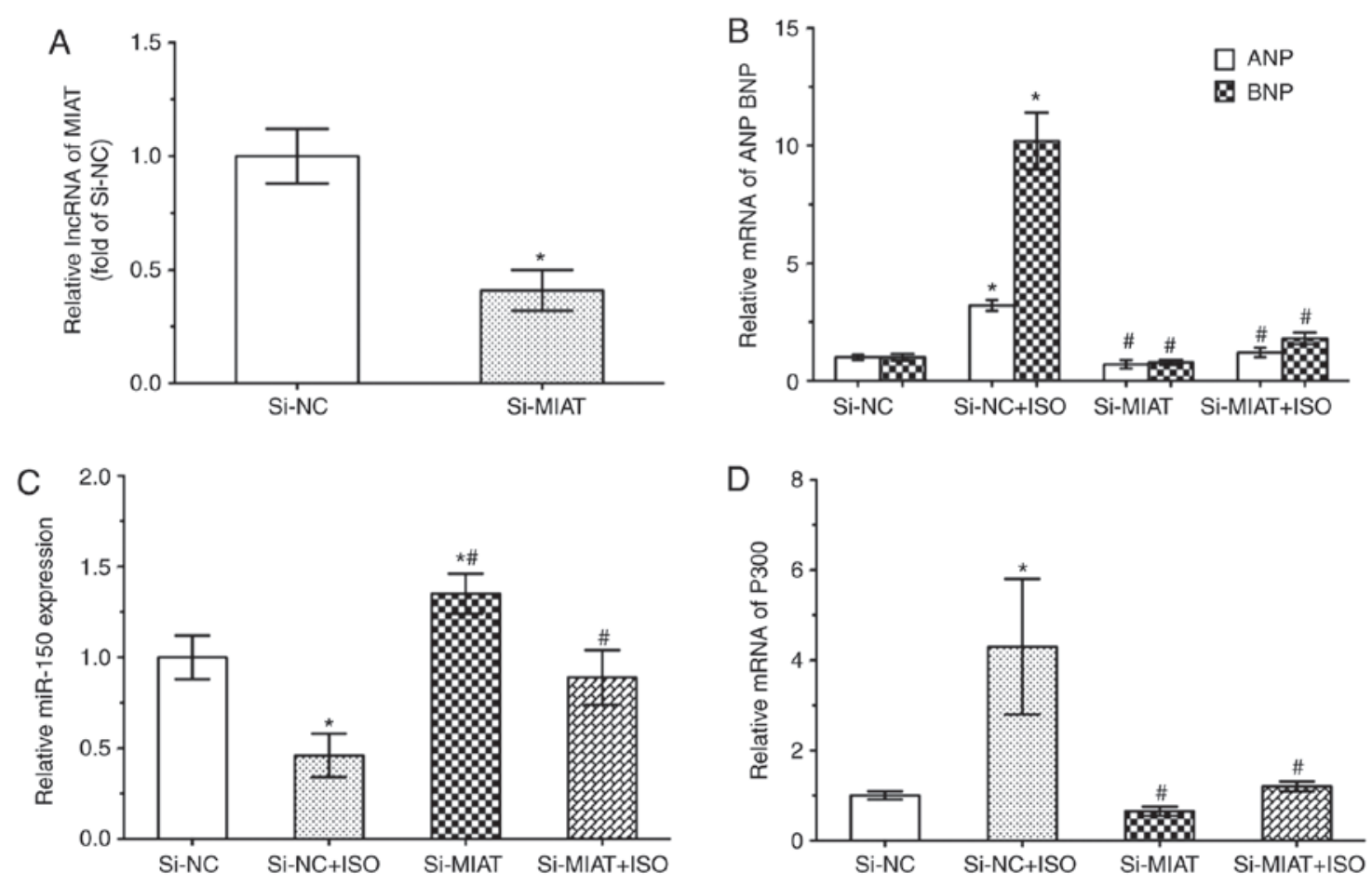

Figure 6. MIAT upregulates P300 expression by suppressing miR-150-5p. NRVMs were transfected with si-MIAT (100 nM) or si-NC (100 nM) for 6 h, and then treated with or without ISO $(10 \mu \mathrm{m})$ for $48 \mathrm{~h}$. Reverse transcription-quantitative polymerase chain reaction revealed the expression levels of (A) MIAT, (B) ANP and BNP expression, (C) miR-150-5p and (D) P300. ${ }^{\mathrm{P}}<0.05$ vs. si-NC group ( $\mathrm{n}=3$ ); ${ }^{*} \mathrm{P}<0.05$ vs. si-NC + ISO group ( $\mathrm{n}=3$ ). ANP, atrial natriuretic peptide; BNP, brain natriuretic peptide; si-, small interfering RNA; miR, microRNA; NC, negative control; ISO, isoproterenol; MIAT, myocardial infarction-associated transcript; miR, microRNA.

miR-150-5p expression was significantly increased in si-MIAT cells compared with in si-NC cells, (Fig. 6C). Similar results with the expression levels of P300 expression (Fig. 6D). These results indicated that MIAT may upregulate the expression of ANP, BNP and P300 expression by suppressing miR-150-5p in NRVMs. 


\section{Discussion}

Previous studies have demonstrated that lncRNAs are correlated with cardiac diseases (19-21); however, few studies have focused on the effect of IncRNAs in cardiac hypertrophy. MIAT is an lncRNA isolated as a candidate gene for myocardial infarction and is expressed predominantly in the heart and brain tissues (8). It has been reported that the abnormal expression of MIAT is involved in cell proliferation, apoptosis and migration in numerous diseases, including in myocardial infarction, microvascular dysfunction and diabetes $(7,22,23)$. However, whether the IncRNA MIAT has a regulatory effect on hypertrophy remains known.

The present study attempted to investigate the effect of MIAT in cardiac hypertrophy and explore the possible underlying mechanisms. The study findings were as follows: i) MIAT was upregulated by ISO, as demonstrated in vivo and in vitro; ii) subsequent to the silencing of MIAT, the expression levels of ANP and BNP decreased in ISO-treated NRVM cardiomyocytes, demonstrating the possible association between MIAT and hypertrophy; and iii) MIAT silencing was associated with increased miR-150-5p and decreased P300 expression levels in hypertrophic NRVMs.

It has been indicated that miR-150 mediates the function of MIAT, and that MIAT may function as a regulator of miR-150 (24). Thus, as expected, MIAT silencing in the current study increased the expression of miR-150-5p in myocytes and decreased P300 expression. Conversely, ISO treatment suppressed miR-150-5p and enhanced P300 expression in NRVMs. These results suggested the involvement of the MIAT/miR-150-5p/P300 network in cardiac hypertrophy; P300 may also be a downstream target of MIAT.

The importance of P300 in the development of cell proliferation and differentiation is well-known (25-27). By binding specifically to phosphorylated CREB protein, P300 mediates the regulation of the cAMP gene $(28,29)$. Previous studies have reported that $\mathrm{P} 300$ contains a bromodomain and is involved in interleukin-6 signaling $(30,31)$. This gene has been identified as potentially serving an important role in the regulation of hypoxia-induced genes $(32,33)$. Furthermore, the P300 protein may activate transcription by binding to transcription factors; thus, P300 is also known as a transcriptional coactivator (34-36). Previous studies have demonstrated that miR-150 regulates the expression of the transcriptional co-activator P300 (37,38). Accordingly, the present study identified that P300 was a downstream target of MIAT/miR-150-5p.

In conclusion, the results of the present study confirmed that the IncRNA MIAT was associated with the miR-150-5p/P300 signaling pathway in cardiac hypertrophy, providing novel insights for understanding the function of lncRNAs in the pathogenesis of cardiac hypertrophy.

\section{Acknowledgements}

Not applicable.

\section{Funding}

This study was supported by grants from the National Key R\&D Program of China (grant no. 2017YFC1307600) and the Natural Science Foundation of Liaoning Province (grant no. 2013021090).

\section{Availability of data and materials}

All data generated or analyzed during this study are included in this published article.

\section{Authors' contributions}

ZL and YL conducted the experiments and wrote the study. XG, GS and QM made substantial contributions to the design of the present study. YD performed data analysis, and produced the figures and tables. GZ conducted animal experiments and wrote the corresponding results of the paper. YS performed cell experiments and wrote the corresponding results of the paper. All authors read and approved the final manuscript.

\section{Ethics approval and consent to participate}

All procedures were performed in accordance with the Ethical Standards of the Committee on Animal Experimentation of China Medical University (project identification code, SCXK-2013-0001; Shenyang, China), and were approved by the Ethical Standards of the Committee on Animal Experimentation of the First Hospital of China Medical University (no. 2017010).

\section{Patient consent for publication}

Not applicable.

\section{Competing interests}

The authors declare that they have no competing interests.

\section{References}

1. Bertels F, Gokhale CS and Traulsen A: Discovering complete quasispecies in bacterial genomes. Genetics 206: 2149-2157, 2017.

2. Wu DD, Irwin DM and Zhang YP: De novo origin of human protein-coding genes. PLoS Genet 7: e1002379, 2011.

3. Zhang D, Xiong M, Xu C, Xiang $\mathrm{P}$ and Zhong X: Long noncoding RNAs: An overview. Methods Mol Biol 1402: 287-295, 2016.

4. Meller VH, Joshi SS and Deshpande N: Modulation of chromatin by noncoding RNA. Annu Rev Genet 49: 673-695, 2015.

5. Tan JY, Vance KW, Varela MA, Sirey T, Watson LM, Curtis HJ, Marinello M, Alves S, Steinkraus BR, Cooper S, et al: Cross-talking noncoding RNAs contribute to cell-specific neurodegeneration in SCA7. Nat Struct Mol Biol 21: 955-961, 2014.

6. Beck ZT, Xing Z and Tran EJ: LncRNAs: Bridging environmental sensing and gene expression. RNA Biol 13: 1189-1196, 2016.

7. Ishii N, Ozaki K, Sato H, Mizuno H, Saito S, Takahashi A, Miyamoto Y, Ikegawa S, Kamatani N, Hori M, et al: Identification of a novel non-coding RNA, MIAT, that confers risk of myocardial infarction. J Hum Genet 51: 1087-1099, 2006.

8. Liao J, He Q, Li M, Chen Y, Liu Y and Wang J: LncRNA MIAT: Myocardial infarction associated and more. Gene 578: 158-161, 2016.

9. Jiang Q, Shan K, Qun-Wang X, Zhou RM, Yang H, Liu C, Li YJ, Yao J, Li XM, Shen Y, et al: Long non-coding RNA-MIAT promotes neurovascular remodeling in the eye and brain. Oncotarget 7: 49688-49698, 2016. 
10. Rao SQ, Hu HL, Ye N, Shen Y and Xu Q: Genetic variants in long non-coding RNA MIAT contribute to risk of paranoid schizophrenia in a Chinese Han population. Schizophr Res 166 : 125-130, 2015.

11. Aprea J, Prenninger S, Dori M, Ghosh T, Monasor LS, Wessendorf E, Zocher S, Massalini S, Alexopoulou D, Lesche M, et al: Transcriptome sequencing during mouse brain development identifies long non-coding RNAs functionally involved in neurogenic commitment. EMBO J 32: 3145-3160, 2013.

12. Ishizuka A, Hasegawa Y, Ishida K, Yanaka K and Nakagawa S: Formation of nuclear bodies by the lncRNA Gomafu-associating proteins Celf3 and SF1. Genes Cells 19: 704-721, 2014.

13. Li Y, Wang J, Sun L and Zhu S: LncRNA myocardial infarction-associated transcript (MIAT) contributed to cardiac hypertrophy by regulating TLR4 via miR-93. Eur J Pharmacol 818: 508-517, 2018.

14. Zhu XH, Yuan YX, Rao SL and Wang P: LncRNA MIAT enhances cardiac hypertrophy partly through sponging miR-150. Eur Rev Med Pharmacol Sci 20: 3653-3660, 2016.

15. Li J, Liu K, Gao X, Yao B, Huo K, Cheng Y, Cheng X, Chen D, Wang B, Sun W, et al: Oxygen- and nitrogen-enriched 3D porous carbon for supercapacitors of high volumetric capacity. ACS Appl Mater Interfaces 7: 24622-24628, 2015.

16. Qu Q, Liu Y, Yan X, Fan X, Liu N and Wu G: A Novel pentapeptide targeting integrin $\beta 3$-subunit inhibits platelet aggregation and its application in rat for thrombosis prevention. Front Pharmacol 7 : 49, 2016.

17. McCommis KS, Douglas DL, Krenz $M$ and Baines CP: Cardiac-specific hexokinase 2 overexpression attenuates hypertrophy by increasing pentose phosphate pathway flux. J Am Heart Assoc 2: e000355, 2013.

18. Livak KJ and Schmittgen TD: Analysis of relative gene expression data using real- time quantitative PCR and the 2(-delta delta C(T)) method. Methods 25: 402-408, 2001.

19. Ounzain S, Micheletti R, Beckmann T, Schroen B, Alexanian M, Pezzuto I, Crippa S, Nemir M, Sarre A, Johnson R, et al: Genome-wide profiling of the cardiac transcriptome after myocardial infarction identifies novel heart-specific long non-coding RNAs. Eur Heart J 36: 353a-368a, 2015

20. Guo X, Chang Q, Pei H, Sun X, Qian X, Tian C and Lin H: Long non-coding RNA-mRNA correlation analysis reveals the potential role of HOTAIR in pathogenesis of sporadic thoracic aortic aneurysm. Eur J Vasc Endovasc Surg 54: 303-314, 2017.

21. Kitow J, Derda AA, Beermann J, Kumarswarmy R, Pfanne A, Fendrich J, Lorenzen JM, Xiao K, Bavendiek U, Bauersachs J and Thum T: Mitochondrial long noncoding RNAs as blood based biomarkers for cardiac remodeling in patients with hypertrophic cardiomyopathy. Am J Physiol Heart Circ Physiol 311: H707-H712, 2016

22. Yan B, Yao J, Liu JY, Li XM, Wang XQ, Li YJ, Tao ZF, Song YC, Chen Q and Jiang Q: IncRNA-MIAT regulates microvascular dysfunction by functioning as a competing endogenous RNA. Circ Res 116: 1143-1156, 2015.

23. Zhang J, Chen M, Chen J, Lin S, Cai D, Chen C and Chen Z: Long non-coding RNA MIAT acts as a biomarker in diabetic retinopathy by absorbing miR-29b and regulating cell apoptosis. Biosci Rep 37: BSR20170036, 2017.

24. Zhang HY, Zheng FS, Yang W and Lu JB: The long non-coding RNA MIAT regulates zinc finger E-box binding homeobox 1 expression by sponging miR-150 and promoteing cell invasion in non-small-cell lung cancer. Gene 633: 61-65, 2017.

25. Otero M,Peng H, Hachem KE, Culley KL, Wondimu EB, Quinn J, Asahara $\mathrm{H}$, Tsuchimochi $\mathrm{K}$, Hashimoto $\mathrm{K}$ and Goldring MB ELF3 modulates type II collagen gene (COL2A1) transcription in chondrocytes by inhibiting SOX9-CBP/p300-driven histone acetyltransferase activity. Connect Tissue Res 58: 15-26, 2017.
26. Tikhanovich I, Zhao J, Bridges B, Kumer S, Roberts B and Weinman SA: Arginine methylation regulates c-Myc-dependent transcription by altering promoter recruitment of the acetyltransferase p300. J Biol Chem 292: 13333-13344, 2017.

27. Clark MD, Kumar GS, Marcum R, Luo Q, Zhang Y and Radhakrishnan I: Molecular basis for the mechanism of constitutive CBP/p300 coactivator recruitment by CRTC1-MAML2 and Its implications in cAMP signaling. Biochemistry 54: 5439-5446, 2015.

28. Naqvi S, Martin KJ and Arthur JS: CREB phosphorylation at Ser133 regulates transcription via distinct mechanisms downstream of cAMP and MAPK signalling. Biochem J 458: 469-479, 2014.

29. Hou T, Ray S, Lee C and Brasier AR: The STAT3 NH2-terminal domain stabilizes enhanceosome assembly by interacting with the p300 bromodomain. J Biol Chem 283: 30725-30734, 2008.

30. Zgheib C, Zouein FA, Chidiac R, Kurdi M and Booz GW: Calyculin A reveals serine/threonine phosphatase protein phosphatase 1 as a regulatory nodal point in canonical signal transducer and activator of transcription 3 signaling of human microvascular endothelial cells. J Interferon Cytokine Res 32: 87-94, 2012.

31. Zhou CH, Zhang XP, Liu F and Wang W: Modeling the interplay between the HIF-1 and p53 pathways in hypoxia. Sci Rep 5: 13834, 2015.

32. Kato H, Tamamizu-Kato S and Shibasaki F: Histone deacetylase 7 associates with hypoxia-inducible factor 1alpha and increases transcriptional activity. J Biol Chem 279: 41966-41974, 2004.

33. Kasper LH, Fukuyama T, Lerach S, Chang Y, Xu W, Wu S, Boyd KL and Brindle PK: Genetic interaction between mutations in c-Myb and the KIX domains of CBP and p300 affects multiple blood cell lineages and influences both gene activation and repression. PloS One 8: e82684, 2013.

34. Wang F, Marshall CB, Yamamoto K, Li GY, Gasmi-Seabrook GM, Okada H, Mak TW and Ikura M: Structures of KIX domain of CBP in complex with two FOXO3a transactivation domains reveal promiscuity and plasticity in coactivator recruitment. Proc Natl Acad Sci USA 109: 6078-6083, 2012.

35. Bedford DC, Kasper LH, Wang R, Chang Y, Green DR and Brindle PK: Disrupting the $\mathrm{CH} 1$ domain structure in the acetyltransferases CBP and p300 results in lean mice with increased metabolic control. Cell Metab 14: 219-230, 2011.

36. Teufel DP, Freund SM, Bycroft M and Fersht AR: Four domains of p300 each bind tightly to a sequence spanning both transactivation subdomains of p53. Proc Natl Acad Sci USA 104: 7009-7014, 2007.

37. Duan Y, Zhou B, Su H, Liu Y and Du C: miR-150-5p regulates high glucose-induced cardiomyocyte hypertrophy by targeting the transcriptional co-activator p300. Exp Cell Res 319: 173-184, 2013.

38. Prakhar P, Holla S, Ghorpade DS, Gilleron M, Puzo G, Udupa V and Balaji KN: Ac2PIM-responsivmiR-150-5p and miR-143 target receptor-interacting protein kinase 2 and transforming growth factor beta-activated kinase 1 to suppress NOD2-induced immunomodulators. J Biol Chem 290: 26576-26586, 2015.

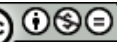

This work is licensed under a Creative Commons Attribution-NonCommercial-NoDerivatives 4.0 International (CC BY-NC-ND 4.0) License. 